\title{
Association of IRS1 Gly972Arg and IRS2 Gly1057Asp polymorphisms with gastric cancer in Turkish subjects
}

\author{
GOKHAN GORGISEN $^{1}$, UGUR KARATAS ${ }^{1}$, CAN ATES $^{2}$, MURAT OKSUZ $^{3}$ and ISMAIL MUSAB GULACAR ${ }^{1}$ \\ Departments of ${ }^{1}$ Medical Biology and ${ }^{2}$ Biostatistics, Faculty of Medicine, Van Yuzuncu Yil University, Van 65080; \\ ${ }^{3}$ Department of Gastroenterology, Private Adana Ortadogu Hospital, Adana 67055, Turkey
}

Received January 29, 2020; Accepted May 14, 2020

DOI: $10.3892 / \mathrm{ol} .2020 .11717$

\begin{abstract}
Insulin receptor substrate (IRS) proteins are cytoplasmic adaptors that transmit the signal from the IR and insulin-like growth factor-1 receptor to effector proteins. Overexpression of IRS proteins has been indicated to be linked to cancer development. In addition to their expression profiles, studies have indicated that polymorphisms of IRS1 and IRS2 are also associated with the susceptibility to numerous cancer types. IRS1 Gly972Arg and IRS2 Gly1057Asp are the common variants of these genes. The present study aimed to determine the association of IRS1 Gly972Arg and IRS2 Gly1057Asp polymorphisms with gastric cancer development. The study included 100 patients with gastric cancer and 100 controls. Single-nucleotide polymorphisms were detected by real-time PCR using Taqman assays. The results suggested that in individuals with the IRS1 Gly/Arg genotype, the odds of having gastric cancer was increased by 7.891 -fold $(95 \%$ CI: 3.251-19.154, $\mathrm{P}<0.001)$ and in individuals with the IRS1 Arg/Arg genotype, it was increased by 22.716 -fold (95\% CI: 6.311-81.761, $\mathrm{P}<0.001)$ compared with those with the IRS1 Gly/Gly genotype. Although the IRS2 Gly1057Asp genotype analysis suggested that subjects with the Asp/Asp genotype had a 2,311-fold increased odds of having gastric cancer compared to those with the Gly/Gly genotype, the result was not statistically significant (95\% CI: 0.800-6.678, $\mathrm{P}=0.122)$. The combined effects of the IRS1 and IRS2 variants on gastric cancer were also determined. The results suggested that individuals with Gly/Arg+Gly/Asp and Gly/Arg+Asp/Asp genotypes had a higher odds of having gastric cancer compared to individuals of the Gly/Gly+Gly/Gly genotype $(\mathrm{P}=0.001$ and $\mathrm{P}=0.027$, respectively). In conclusion, the present results suggested that the IRS1 Gly972Arg and IRS2 Gly1057Asp variations may be associated with an increased susceptibility
\end{abstract}

Correspondence to: Dr Gokhan Gorgisen, Department of Medical Biology, Faculty of Medicine, Van Yuzuncu Yil University, 2 Bardakci Street, Tusba,Van 65080, Turkey

E-mail: gokhangorgisen@gmail.com

Key words: insulin receptor substrate 1, insulin receptor substrate 2, polymorphism, gastric cancer to develop gastric cancer. Further studies with larger sample sizes are required to support the present results and to explore the use of these variations as a biomarker for gastric cancer.

\section{Introduction}

Gastric cancer is the third leading cause of cancer-associated mortality worldwide. It frequently remains undetected until it has reached the advanced stage and the 5-year survival rate is $<20 \%$. Genetic alterations, Helicobacter pylori (H. pylori) infection, obesity, dietary habits and an unhealthy lifestyle are highly linked to gastric cancer (1).

Type 2 diabetes is a common endocrine disorder. Epidemiological studies have indicated a marked association between diabetes and cancer development (2,3). Abnormal glucose homeostasis is a common risk factor for the development of type 2 diabetes and gastric cancer. Although various studies have indicated that diabetes increased the risk of gastric cancer in males, the association between gastric cancer and diabetes remains controversial (4).

Glucose homeostasis is controlled by insulin signaling in the cell. Insulin receptor substrate (IRS) proteins are the major cytoplasmic molecules that regulate insulin signaling and have a pivotal role in the metabolic and mitogenic actions of insulin and insulin-like growth factors (IGFs) (5). IRS1 and IRS2 are the prominent members of this family and are widely expressed in human tissues. Activation of IRS proteins triggers intracellular signaling cascades, which induce the activation of PI3K/AKT and ERK (6). Although they are identified as IRSs, they are able to interact with IGF receptor, leptin, vascular endothelial growth factors, growth hormone, prolactin, integrin, cytokine and interferon receptors. These interactions demonstrated the importance of IRS proteins in cancer development (7). In line with this, overexpression of IRS1 and IRS2 has been reported in numerous cancer cell types (8). In addition to expression and activation profiles, genomic changes of IRS genes also have a critical role in cancer development. In a previous study by our group, novel mutations in the IRS1 gene were detected in patients with non-small cell lung cancer, and they were indicated to have an important influence on the phenotype of lung cancer (9).

IRS1 Gly972Arg (rs1801278) and IRS2 Gly1057Asp (rs1805097) are the most common variants of IRS genes (10). Although numerous studies reported that these variants 
significantly influence the susceptibility to insulin resistance, obesity, polycystic ovary syndrome (PCOS), type 2 diabetes and various cancer types, the results of different studies remain contradictory depending on ethnicity, sample size and cancer type (10-14). Therefore, population-based studies are important to detect the real effect of these variations in cancer development.

The present study aimed to determine the combined effects of IRS1 Gly972Arg (rs1801278) and IRS2 Gly1057Asp (rs1805097) polymorphisms on the susceptibility to gastric cancer in Turkish subjects.

\section{Materials and methods}

Study subjects. The present study was performed on 200 individuals, comprising 100 patients with gastric adenocarcinoma and 100 healthy subjects. In the control group, the subjects had no family history of gastric cancer in the first and second degree and were matched to the cases by age, gender and region. Exclusion criteria for the patient group were receiving chemo- and radiotherapy treatments previously, metabolic disorders including diabetes and obesity, other malignancies and having tumors at a site other than the stomach or a non-adenocarcinoma type of gastric cancer. All patients were diagnosed with gastric adenocarcinoma. Informed written consent was obtained from each subject included and the present study conformed to the standards of the Declaration of Helsinki. The present study was approved by the Ethical Committee of Van Yuzuncu Yil University (Van, Turkey).

DNA extraction and single-nucleotide polymorphism (SNP) genotyping. Genomic DNA was isolated from peripheral blood mononuclear cells using a GF-1 Blood DNA Extraction kit (Vivantis Technologies) according to the manufacturer's protocol. SNPs were analyzed by Real-time PCR technology. Taqman assays (Thermo Fisher Scientific, Inc.) were used for the genotyping of the IRS1 gene Gly972Arg variant (rs1801278; assay ID, C_2384392_20) and the IRS2 gene Gly1057Asp variant (rs1805097; assay ID, C_144336620). Real-time PCR amplification was performed in a $25-\mu 1$ mixture containing 2, $25 \mu \mathrm{l}$ genomic DNA solution (3-20 ng in distilled water), 12, $50 \mu \mathrm{l}$ of $2 \mathrm{X}$ Taqpath ProAmp Master Mix (2X) and 1,25 $\mu \mathrm{l}$ of 20X Taqman SNP Assay mix. SNP genotyping reaction was performed in a 96 well-optical plate on a StepOnePlus System (Thermo Fisher Scientific, Inc.) using the following thermocycling conditions: Initial denaturation of $95^{\circ} \mathrm{C}$ for $10 \mathrm{~min}$, followed by 40 cycles of denaturation at $95^{\circ} \mathrm{C}$ for $15 \mathrm{sec}$ and annealing/extension at $60^{\circ} \mathrm{C}$ for $1 \mathrm{~min}$. The alleles were labeled with fluorescent probes $\mathrm{VIC}^{\circledR}$-dye and FAM ${ }^{\mathrm{TM}}$ dye (Thermo Fisher Scientific, Inc.).

Statistical analysis. Kolmogorov-Smirnov and Shapiro-Wilk tests were used to test for normality of distribution. According to the results, non-parametric tests were preferred. Differences in proportions between groups were assessed by using a $\chi^{2}$ or Fisher's exact test, as appropriate. Continuous variables were compared among categories of grouping variables using the Mann-Whitney U test. To assess the agreement between genotypes observed and those predicted by the Hardy-Weinberg equilibrium, the likelihood ratio test ( $\mathrm{G}$ statistic) was used. In order to define independent risk factors of gastric cancer, multivariate logistic regression analysis was used and adjusted for age and sex, odds ratios were calculated. $\mathrm{P}<0.05$ was considered to indicate a statistically significant difference. Values are expressed as $\mathrm{n}(\%)$, mean \pm standard deviation (SD) or the median (minimum-maximum). For statistical analyses, SPSS software v15.0 (SPSS, Inc.) was used.

\section{Results}

Demographic features of study group. The demographic features of the patients and controls are summarized in Table I. The mean age was $62.65 \pm 13.74$ years in the patient group and $58.78 \pm 13.81$ years in the control group. There was no significant difference in the age and gender distribution between the two groups $(\mathrm{P}>0.005)$. The $\chi^{2}$ test was used to test for the Hardy-Weinberg equilibrium and P-values were determined as $<0.001$ for IRS1 Gly972Arg and 0.0896 for IRS2 Gly1057Asp.

Genotype and allelic frequencies of subjects. Genotype and allelic frequencies were calculated after SNP profiling. Considering the controls separately from the patients with gastric cancer, the genotype frequency of IRS1 Gly972Arg was $91 \%$ for Gly/Gly and 9\% for Gly/Arg, while none of the controls was of the Arg/Arg genotype (Table II). In the patients with gastric cancer, the frequencies of the Gly/Gly, Gly/Arg and Arg/Arg genotypes were 45, 26 and 29\%, respectively (Table II). It was observed that the Gly/Arg and Arg/Arg genotypes were more common in the cancer group compared to the control group. The Gly/Gly genotype was used as a non-exposed reference and logistic regression analyses indicated that individuals with the IRS1 Gly/Arg genotype had a 7.891-fold (95\% CI: 3.251-19.154, $\mathrm{P}<0.001)$ and individuals with the IRS1 Arg/Arg genotype had a 22.716-fold (95\% CI: 6.311-81.761, $\mathrm{P}<0.001)$ higher odds of having gastric cancer compared with those of the IRS1 Gly/Gly genotype (Table II). According to the result of the IRS2 Gly1057Asp genotyping, the frequencies of Gly/Gly, Gly/Asp and Asp/Asp genotypes were 60,33 and $7 \%$, respectively, in the control group and in 57, 33 and $10 \%$, respectively, in the gastric cancer group (Table II). Although subjects with the Asp/Asp genotype had a 2,311-fold increased odds of having gastric cancer compared with those of the Gly/Gly genotype, the result was not statistically significant (95\% CI: 0.800-6.678, $\mathrm{P}=0.122$; Table II).

Logistic regression analyses performed to examine the combined effect of IRS1 Gly972Arg and IRS2 Gly1057Asp variants in gastric cancer revealed that individuals with the Gly/Arg+Gly/Asp genotype had an 8.704-fold and individuals with the Gly/Arg+Asp/Asp genotype had an 11.947-fold increased odds of having gastric cancer compared to individuals with the Gly/Gly+Gly/Gly genotype (95\% CI: 2.303-32.890, $\mathrm{P}=0.001$; 95\% CI: $1.327-107.567, \mathrm{P}=0.027$; Table II). However, no statistically significant difference between the Arg/Arg+Gly/Asp and Gly/Gly+Gly/Gly genotype and the Arg/Arg+Asp/Asp and Gly/Gly+Gly/Gly in terms of the odds of gastric cancer was observed, while a 5.157- and 4.448-fold increased odds of gastric cancer compared to the Gly/Gly+Gly/Gly genotype, respectively, was determined (95\% CI: 0.510-52.114, $\mathrm{P}=0.165$; 95\% CI: 0.440-44.909, P=0.206; Table II).

Determination of allelic frequencies of IRS1 Gly972Arg indicated that in the control group, all subjects carried the Gly 
Table I. Demographic features of patients and controls.

\begin{tabular}{lcc}
\hline Variable & Patients $(\mathrm{n}=100)$ & Control $(\mathrm{n}=100)$ \\
\hline Age (years) & & \\
Mean \pm SD & $62.65 \pm 13.74$ & $58.78 \pm 13.81$ \\
Median (minimum-maximum) & $62.0(26.0-87.0)$ & $60.0(24.0-85.0)$ \\
$<60$ & $36(40.9)$ & $52(59.1)$ \\
$>60$ & $42(46.7)$ & $48(53.3)$ \\
Sex & & 0.063 \\
Male & $53(52.0)$ & $49(48.0)$ \\
Female & $47(48.0)$ & $51(52.0)$ \\
\hline
\end{tabular}

Values are expressed as n (\%) unless otherwise specified. SD, standard deviation.

Table II. Genotype distributions of IRS1 and IRS2 alleles and possible combinations in patients with gastric cancer and controls.

\begin{tabular}{|c|c|c|c|c|c|}
\hline Mutation & Control & Patients & P-value ${ }^{a}$ & $\mathrm{OR}^{\mathrm{c}}(95 \% \mathrm{CI})$ & $\mathrm{P}$-value ${ }^{\mathrm{b}}$ \\
\hline IRS1 Gly972Arg & & & $<0.001$ & & \\
\hline Gly/Gly & $91(91)$ & $45(45)$ & & 1 (reference) & \\
\hline Gly/Arg & $9(9)$ & $26(26)$ & & $7.891(3.251-19.154)$ & $<0.001$ \\
\hline Arg/Arg & $0(0)$ & $29(29)$ & & $22.716(6.311-81.761)$ & $<0.001$ \\
\hline IRS2 Gly1057Asp & & & 0.738 & & \\
\hline Gly/Gly & $60(60)$ & $57(57)$ & & 1 (reference) & \\
\hline Gly/Asp & $33(33)$ & $33(33)$ & & $1.406(0.725-2.727)$ & 0.313 \\
\hline Asp/Asp & $7(7)$ & $10(10)$ & & $2.311(0.800-6.678)$ & 0.122 \\
\hline \multicolumn{6}{|l|}{ IRS1 and IRS2 } \\
\hline Gly/Gly+Gly/Gly & 29 & 55 & & 1 (reference) & \\
\hline Gly/Arg+Gly/Asp & 15 & 30 & & $8.704(2.303-32.890)$ & 0.001 \\
\hline Gly/Arg+Asp/Asp & 5 & 1 & & 11.947 (1.327-107.567) & 0.027 \\
\hline Arg/Arg+Gly/Asp & 4 & 0 & & $5.157(0.510-52.114)$ & 0.165 \\
\hline $\mathrm{Arg} / \mathrm{Arg}+\mathrm{Asp} / \mathrm{Asp}$ & 3 & 0 & & $4.448(0.440-44.909)$ & 0.206 \\
\hline
\end{tabular}

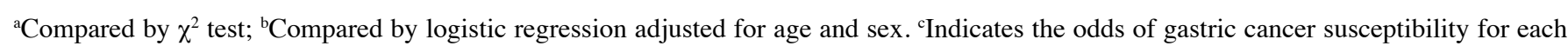
genotype compared to the reference genotype indicated by ' 1 '. Values are expressed as n (\%). OR, odds ratio; IRS1, insulin receptor substrate 1 .

Table III. Allelic frequencies of IRS1 and IRS2 variants in gastric cancer patients and controls.

\begin{tabular}{|c|c|c|c|c|c|}
\hline Mutation/inclusion of 'Gly' & Patients & Control & P-value ${ }^{a}$ & OR (\%95 CI) & P-value ${ }^{b}$ \\
\hline IRS1 Gly972Arg & & & $<0.001$ & & \\
\hline Gly included & $71(71)$ & $100(100)$ & & 1 & \\
\hline Gly not included & $29(29)$ & $0(0)$ & & $\ldots(0.0-\ldots)$ & $\ldots$ \\
\hline IRS2 Gly1057Asp & & & 0.447 & & \\
\hline Gly included & $90(90)$ & $93(93)$ & & 1 & 0.449 \\
\hline Gly not included & $10(10)$ & $7(7)$ & & $1.476(0.539-4.047)$ & \\
\hline
\end{tabular}

${ }^{\mathrm{a} C o m p a r e d ~ b y ~} \chi^{2}$ test; ${ }^{\mathrm{b} C o m p a r e d ~ b y ~ l o g i s t i c ~ r e g r e s s i o n ~ a d j u s t e d ~ f o r ~ a g e ~ a n d ~ s e x . ~ V a l u e s ~ a r e ~ e x p r e s s e d ~ a s ~ n ~(\%) . ~ O R, ~ o d d s ~ r a t i o ; ~ I R S 1, ~ i n s u l i n ~}$ receptor substrate 1 .

allele, whereas the Gly allele frequency was $71 \%$ in the patient group. While the Arg allele was not detected in the controls, $29 \%$ of the patients with gastric cancer had the Arg allele (Table III). The allelic frequency of IRS1 Arg972 was significantly higher in 
patients with gastric cancer compared to the controls. However, no significant difference was observed between patients and controls in allelic distributions of IRS2 variants (Table III).

\section{Discussion}

IRS1 and IRS2 are common mediators of cancer progression and metastasis. Overexpression of IRS1 and IRS2 is associated with proliferation and metastasis in numerous cancer types. In addition to their expression profiles, genomic variations of these genes have been linked to the development of cancer $(9,15)$.

IRS genes have evolutionarily conserved sequences. IRS1 Gly972 and IRS2 Gly1057 are located near PI3K-binding sites of proteins and they represent the same position in the structure of proteins $(16,17)$. Therefore, understanding the combined effects of these polymorphisms in the development of diseases is important. The association of IRS1 Gly972Arg (rs1801278) and IRS2 Gly1057Asp (rs1805097) polymorphisms with gastric cancer has not been previously investigated and, to the best of our knowledge, the present study was the first to assess this in a Turkish population.

Previous studies have focused on the effects of IRS1 Gly972Arg (rs1801278) and IRS2 Gly1057Asp (rs1805097) variants on metabolic diseases and functional studies suggested that IRS1 Gly972Arg causes conformational changes in the IRS1 and IRS2 proteins, leading to a decreased binding efficiency of PI3K and activation of AKT $(18,19)$.

To date, only a limited number of studies have examined the effects of these polymorphisms on cancer as opposed to the vast literature on their implication in metabolic diseases. The present study indicated that the susceptibility of individuals with the Gly/Arg genotype in the IRS1 Gly972Arg variant to develop gastric cancer was 7.891-fold increased and that of individuals with the Arg/Arg genotype was 22.716-fold increased as compared with that of individuals with the Gly/Gly genotype. In concordance with the present results, the study by Li et al (20) from 2014 assessing the Gly972Arg variant in 3,708 cases of colorectal cancer suggested that this variant was had a significant role in the development of colorectal cancer in mixed populations. A meta-analysis study by Zhang et al (12), also revealed a significant association of the IRS1 Gly972Arg polymorphism with breast and ovarian cancer. Although Fall et al (21) reported that the Arg/Arg genotype was associated with a two-fold increase in the risk of developing prostate cancer, another study indicated no such link (22). These contradictory results may due to differences in ethnicity, sample size and cancer type.

A study on Turkish subjects indicated that the variant allele of Asp1057 of IRS2 was associated with endometrial cancer, but they did not identify any influence on ovarian cancer $(23,24)$. Another study in a Turkish population indicated that the IRS2 Gly1057Asp variant was not associated with colorectal cancer (25). On the other hand, a recent meta-analysis study indicated that the Asp1057 variant was associated with a decreased risk of colorectal cancer (26). Another meta-analysis study failed to reveal any link of the Asp1057 variant with colorectal and breast cancer (27). The present study did not find any association between Asp1057 and gastric cancer and these results were conistent with those of previous studies. However, while it was observed that the gastric cancer risk in individuals with the Asp/Asp genotype was 2.311-fold of that in individuals with the Gly/Gly genotype, this was not statistically significant.

While no previous study appears to have examined the combined effects of two variants in cancer, certain studies indicated their interaction with metabolic disorders, including type 2 diabetes and PCOS. Lin et al (10), revealed a significant association of IRS1 Arg972 and IRS2 1057Asp variants with PCOS in a Chinese population. A study on Punjabi Pakistani subjects also observed a higher combined minor genotype frequency in patients with type 2 diabetes compared to controls (28). In line with the results of those previous studies, the present study revealed that combined effects of these variants also had a significant influence on the development of gastric cancer in the present population.

Of note, the present study had certain limitations. In part due to limited funding, the sample size was relatively small, and therefore, the results require further validation in larger sample groups and a meta-analysis. As another limitation, the associations between IRS1 and IRS2 variants, $H$. pylori infection and histopathological subtypes of gastric cancer were not examined. The present results should be considered as preliminary to future studies and the establishment of new hypotheses. In spite of those limitations, the present study provided evidence for the presence of a significant association between the IRS1 gene and gastric cancer development.

In conclusion, the present study determined a significant association of variants of the IRS1 gene, as well as the interaction of IRS1 and IRS2 gene polymorphisms, with gastric cancer. The importance of considering combined effects of SNPs in the pathogenesis of diseases was showcased. To the best of our knowledge, the present study was the first to preliminarily examine the separate and combined effects of IRS1 and IRS2 gene variants on gastric cancer susceptibility. Further studies with larger sample size, different ethnic groups and populations are required to verify the present results and define the exact roles of two variations in the predisposition for gastric cancer.

\section{Acknowledgements}

Not applicable.

\section{Funding}

This study was supported by a grant from The Scientific Research Projects Coordination Unit of Van Yuzuncu Yil University (Van, Turkey; project no. TYL 2019-8109).

\section{Availability of data and materials}

The datasets used and/or analyzed during the present study are available from the corresponding author on reasonable request.

\section{Authors' contributions}

GG was responsible for the study conception and design of the experiments, as well as data analysis, and drafted and finalized the manuscript. UK and IMG performed all experiments. MO collected the specimens and CA was responsible for the design of the study and statistical analyses. All authors have read and approved the final manuscript. 


\section{Ethics approval and consent to participate}

Informed written consent was obtained from each participant and the study conformed to the standards of the Declaration of Helsinki. This study was approved by the Ethics Committee of Van Yuzuncu Yil University (Van, Turkey; approval date: 09/11/2018; No. 08).

\section{Patient consent for publication}

Not applicable.

\section{Competing interests}

The authors declare that they have no competing interests.

\section{References}

1. Rawla P and Barsouk A: Epidemiology of gastric cancer: Global trends, risk factors and prevention. Prz Gastroenterol 14: 26-38, 2019.

2. Giovannucci E, Harlan DM, Archer MC, Bergenstal RM, Gapstur SM, Habel LA, Pollak M, Regensteiner JG and Yee D: Diabetes and cancer: A consensus report. Diabetes Care 33: 1674-1685, 2010.

3. Saarela K, Tuomilehto J, Sund R, Keskimäki I, Hartikainen S and Pukkala E: Cancer incidence among Finnish people with type 2 diabetes during 1989-2014. Eur J Epidemiol 34: 259-265, 2019.

4. Abudawood M: Diabetes and cancer: A comprehensive review. J Res Med Sci 24: 94, 2019.

5. Gorgisen G, Gulacar IM and Ozes ON: The role of insulin receptor substrate (IRS) proteins in oncogenic transformation Cell Mol Biol (Noisy-le-grand) 63: 1-5, 2017.

6. Shaw LM: The insulin receptor substrate (IRS) proteins: At the intersection of metabolism and cancer. Cell Cycle 10: 1750-1756, 2011.

7. Gibson SL, Ma Z, and Shaw LM: Divergent roles for IRS-1 and IRS-2 in breast cancer metastasis. Cell Cycle 6: 631-637, 2007.

8. Mardilovich K, Pankratz SL and Shaw LM: Expression and function of the insulin receptor substrate proteins in cancer. Cell Commun Signal 7: 14, 2009.

9. Gorgisen G, Hapil FZ, Yilmaz O, Cetin Z, Pehlivanoglu S, Ozbudak IH, Erdogan A and Ozes ON: Identification of novel mutations of Insulin Receptor Substrate 1 (IRS1) in tumor samples of non-small cell lung cancer (NSCLC): Implications for aberrant insulin signaling in development of cancer. Genet Mol Bio 42: 15-25, 2019.

10. Lin MW, Huang MF and Wu MH: Association of Gly972Arg variant of insulin receptor subtrate-1 and Gly1057Asp variant of insulin receptor subtrate- 2 with polycystic ovary syndrome in the Chinese population. J Ovarian Res 7: 92, 2014.

11. Gelaleti RB, Damasceno DC, Salvadori DM, Marcondes JP, Lima PH, Morceli G, Calderon IM and Rudge MV: IRS-1 gene polymorphism and DNA damage in pregnant women with diabetes or mild gestational hyperglycemia. Diabetol Metab Syndr 7: 30, 2015

12. Zhang $\mathrm{H}$, Wang $\mathrm{A}, \mathrm{Ma} \mathrm{H}$ and $\mathrm{Xu} \mathrm{Y}$ : Association between insulin receptor substrate 1 Gly972Arg polymorphism and cancer risk. Tumour Biol 34: 2929-2936, 2013.
13. Ayaz L, Karakaş Çelik S and Cayan F: The G1057D polymorphism of insulin receptor substrate-2 associated with gestational diabetes mellitus. Gynecol Endocrinol 30: 165-168, 2014.

14. Christopoulos P, Mastorakos G, Gazouli M, Deligeoroglou E, Katsikis I, Diamanti-Kandarakis E, Panidis D, Creatsas G: Study of association of IRS-1 and IRS-2 genes polymorphisms with clinical and metabolic features in women with polycystic ovary syndrome. Is there an impact? Gynecol Endocrinol 26: 698-703, 2010.

15. Carvalheira JB, Ribeiro EB, Folli F, Velloso LA and Saad MJ: Interaction between leptin and insulin signaling pathways differentially affects JAK-STAT and PI 3-kinase-mediated signaling in rat liver. Biol Chem 384: 151-159, 2003.

16. D'Alfonso R, Marini MA, Frittitta L, Sorge R, Frontoni S, Porzio O, Mariani LM, Lauro D, Gambardella S, Trischitta V, et al: Polymorphisms of the insulin receptor substrate- 2 in patients with type 2 diabetes. J Clin Endocrinol Metab 88: 317-322, 2003.

17. Arikoglu H, Aksoy Hepdogru M, Erkoc Kaya D, Asik A, Ipekci SH and Iscioglu F: IRS1 gene polymorphisms Gly972Arg and Ala513Pro are not associated with insulin resistance and type 2 diabetes risk in non-obese Turkish population. Meta Gene 2: 579-585, 2014.

18. Brancati FL, Kao WL, Folsom AR, Watson RL and Szklo M: Incident type 2 diabetes mellitus in African American and white adults: The atherosclerosis risk in communities study. JAMA 283: 2253-2259, 2000.

19. Thameem F, Puppala S, Schneider J, Bhandari B, Arya R, Arar NH, Vasylyeva TL, Farook VS, Fowler S, Almasy L, et al: The Gly(972)Arg variant of human IRS1 gene is associated with variation in glomerular filtration rate likely through impaired insulin receptor signaling. Diabetes 61: 2385-2393, 2012.

20. Li P, Wang L, Liu L, Jiang H, Ma C, Hao T: Association between IRS-1 Gly972Arg polymorphism and colorectal cancer risk. Tumour Biol 35: 6581-6585, 2014.

21. Fall K, Stark JR, Mucci LA, Chan J, Stampfer MJ, Kurth T, Febbo PG, Kantoff P and Ma J: No association between a polymorphic variant of the IRS-1 gene and prostate cancer risk. Prostate 68: 1416-1420, 2008.

22. Neuhausen SL, Slattery ML, Garner CP, Ding YC, Hoffman M and Brothman AR: Prostate cancer risk and IRS1, IRS2, IGF1, and INS polymorphisms: Strong association of IRS1 G972R variant and cancer risk. Prostate 64: 168-174, 2005.

23. Cayan F, Tok E, Aras-Ateş N, Ayaz L, Akbay E, Gen R, Karakaş S and Dilek S: Insulin receptor substrate 2 gene polymorphism: Is it associated with endometrial cancer? Gynecol Endocrinol 26: 378-382, 2010.

24. Çayan F, Dilek S, Ramazan G, Akbay E, Aras-Ates N and Ayaz L: Association of insulin receptor substrate- 2 gene polymorphism with ovarian cancer. Balkan Med J 28: 14-17, 2011.

25. Yukseloglu EH, Celik SK, Kucuk MU, Yalin E, Ozkal SS, Ates C, Berkoz M, Yalin S and Ates NA: IRS-2 G1057D polymorphism in Turkish patients with colorectal cancer. Prz Gastroenterol 9: 88-92, 2014.

26. Yin J, Zhang Z, Zheng H and Xu L: IRS-2 rs1805097 polymorphism is associated with the decreased risk of colorectal cancer. Oncotarget 8: 25107-25114, 2017.

27. Hu Y, Zhou M, Zhang K, Kong X, Hu X, Li K and Liu L: Lack of association between insulin receptor substrate2 rs1805097 polymorphism and the risk of colorectal and breast cancer: A meta-analysis. PLoS One 9: e86911, 2014.

28. Ijaz A, Babar S, Sarwar S, Shahid SU and Shabana: The combined role of allelic variants of IRS-1 and IRS-2 genes in susceptibility to type 2 diabetes in the Punjabi Pakistani subjects. Diabetol Metab Syndr 11: 64, 2019. 